\title{
Dual fluorescence of 9-anthryl-substituted oligothiophenes in nonpolar environment
}

\author{
P. Emele, D.U. Meyer, N. Holl, H. Port, H.C. Wolf \\ 3. Physikalisches Institut, Universität Stuttgart, Stuttgart, Germany \\ F. Würthner, P. Bäuerle and F. Effenberger
Institut für Organische Chemie, Universität Stuttgart, Stuttgart, Germany
}

Received 5 August 1993; in final form 28 December 1993

\begin{abstract}
9-Anthryl-oligothiophenes (9A-T,$n=1-4$ ) have been studied in $n$-hexane solution between helium and room temperature using absorption, fluorescence emission and excitation spectra and time resolved fluorescence measurements. These compounds are reference systems for studies on intramolecular energy and charge transfer in donor/acceptor-substituted conjugated chain molecules. The absorption spectra show contributions of both substituents anthracene and oligothiophene, but no additional bands due to mixed electronic states. Dual fluorescence is observed at $T \geqslant 120 \mathrm{~K}$ for the compounds $9 \mathrm{~A}-\mathrm{T}_{n}(n=1-3)$, but not for

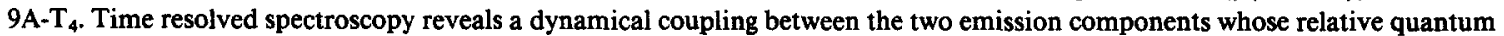
yields are strongly temperature dependent. The occurrence of the dual fluorescence is explained by an intramolecular torsional motion between the two molecular subunits.
\end{abstract}

\section{Introduction}

Bichromophoric systems containing anthracene as one molecular subunit have obtained much interest for photophysical studies. Most work has been focused on systems with little degree of $\pi$-resonance between the two chromophores for the ground as well as the excited state, e.g. 9-phenylanthracene $[1,2]$, 9(2-naphthyl)-anthracene [1] and 9-9'-bianthryl [15] due to the sterical hindrance which counteracts the planarization of the molecules. In these systems the variation of the $\pi$-system changes also the sterical hindrance between the subunits.

In our group polyenes with systematic variation of the chain length terminally substituted with anthracene were investigated [6]. Here the variation of the $\pi$-system takes place without change of the sterical hindrance. In terminally substituted polyene systems containing anthracene and porphyrin endgroups intramolecular energy transfer from anthracene to porphyrin is observed $[7,8]$.

Due to possible photoinduced isomerization and considerable radiationless deactivation rates on the conjugated chain the polyene system seems to be less suitable for further applications [9]. Therefore we have introduced oligothiophenes as alternative chain molecules. Oligothiophenes are stable compounds with high fluorescence quantum yields [10] which have also a large degree of $\pi$-delocalization along the chain. By stepwise extension of the chain by oligothiophene units the conjugated $\pi$-system is enlarged by two double bonds. In this paper we report on the optical properties of 9-anthryl-substituted oligothiophenes as constituents for supermolecular systems allowing for intramolecular energy transfer.

\section{Experimental}

The preparation of 9-anthryl-oligothiophenes 9A$\mathrm{T}_{n}$ (scheme 1) involves nickel-catalyzed cross-coupling reactions of metallated 5-bromo- $\alpha$-oligothiophenes [11] with 9-bromo-anthracene or with 2-(9anthryl)-5-bromothiophenes. All compounds $9 \mathrm{~A}-\mathrm{T}_{n}$ were extensively purified using column chromatography, MPLC and recrystallization, and have been 


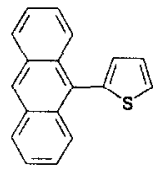

$9 A-T_{1}$

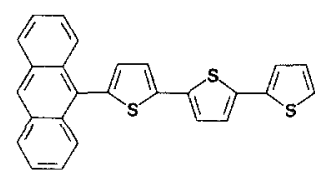

$9 \mathrm{~A}_{3}$

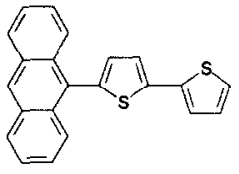

$9 \mathrm{~A}-\mathrm{T}_{2}$

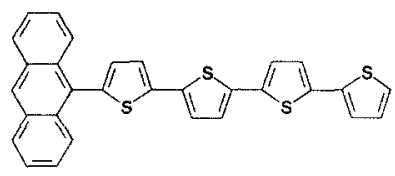

$9 \mathrm{~A} \cdot \mathrm{T}_{4}$
Scheme 1. Molecular structure of the 9-anthryl-substituted oligothiophenes, 9A-T with $n=1-4$.

characterized by ${ }^{1} \mathrm{H}$ - and ${ }^{13} \mathrm{C}-\mathrm{NMR}$ spectra and correct elemental analyses [12].

Optical measurements were performed in liquid $n$ hexane solutions kept at concentrations of $10^{-6} . . .10^{-5}$ $\mathrm{mol} / \mathrm{l}$ in order to maintain low optical densities and to exclude intermolecular interactions. $n$-Hexane (Uvasol, Merck) was used as purchased. A Perkin Elmer Lambda $16 \mathrm{UV} / \mathrm{VIS}$ spectrometer was applied for recording of the optical absorption spectra at $T=295 \mathrm{~K}$. For fluorescence, fluorescence excitation and time resolved fluorescence measurements the solutions were degassed from oxygen by repeated freeze-pump-thaw cycles.

The cw emission and excitation spectra were measured using a $450 \mathrm{~W}$ xenon lamp combined with a $0.25 \mathrm{~m}$ double monochromator as light source (bandwidth $2 \mathrm{~nm}$ fwhm) and a $1 \mathrm{~m}$ double monochromator (bandwidth $1 \mathrm{~nm}$ fwhm) with cooled photomultiplier as detection apparatus in photon counting mode. The time resolved emission spectroscopy and the recording of the transients have been done using time correlated single-photon counting after ps laser excitation at $27300 \mathrm{~cm}^{-1}$. By means of fitting the transients deconvoluted with the response of the apparatus the time resolution is about $10 \mathrm{ps.A}$ detailed description is given in [13]. All emission and excitation spectra were corrected for the spectral responses of the experimental setup.

Quantum yields were obtained relative to a common standard, anthracene in $n$-hexane $\left(\Phi_{\mathrm{f}}=0.30\right.$ at $T=295 \mathrm{~K}$ [14]).

\section{Results}

The absorption and fluorescence emission spectra of anthracene and 9-anthryl-oligothiophenes, 9A- $T_{n}$ $(n=1-4)$ at room temperature, are shown in fig. 1. The absorption spectra are composed of three main parts. In the UV region at about $39500 \mathrm{~cm}^{-1}$ the dominant absorption band is seen, which is characteristic for the anthracene $S_{0}-S_{3}$ transition. Evaluation of the integral absorption of this band results in a constant integral intensity with increasing chain length whereas the maximum extinction coefficient decreases. Between 26000 and $32000 \mathrm{~cm}^{-1}$ all compounds show the vibronically resolved anthracene $S_{0^{-}}$ $\mathrm{S}_{1}$ absorption. For $n \geqslant 2$ additional unstructured absorption bands appear, which are shifting monotonically with increasing $n$. These absorption bands correspond to spectral contributions of the oligothiophenes, whose spectra are given as a reference in fig. 1 (in agreement with literature [10]), but

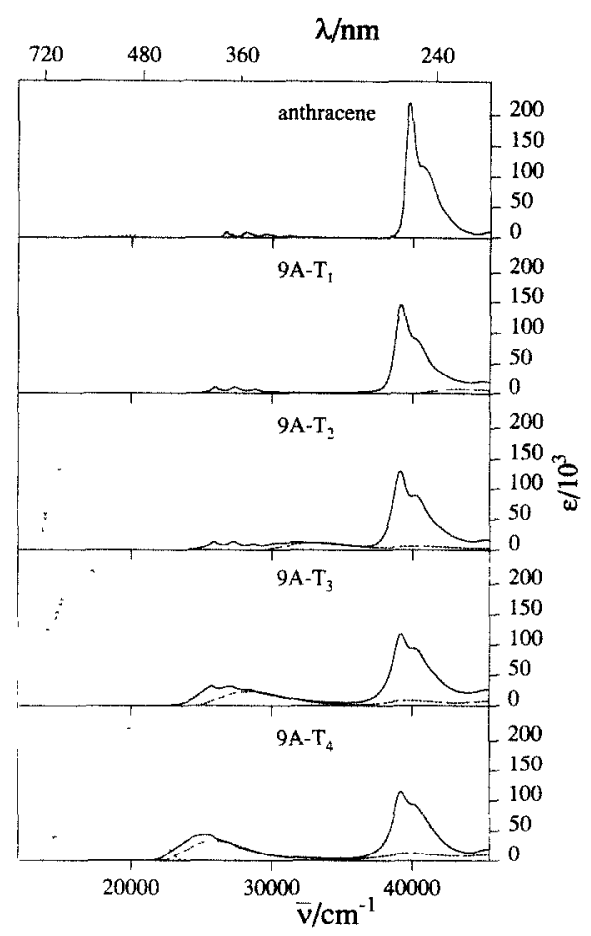

Fig. 1. Spectra of $9 \mathrm{~A}-\mathrm{T}_{n}$ in $n$-hexane at $T=295 \mathrm{~K}:(-)$ absorption, ( $\cdots$ ) fluorescence, normalized to the same maximum intensity, $\lambda_{e x}=257 \mathrm{~nm} .(---)$ Absorption of $T_{n}(n=1-4)$, for comparison. 
are displaced systematically to the red by about 1200 $\mathrm{cm}^{-1}$.

At room temperature the fluorescence emission spectra of all anthryl-oligothiophenes (fig. 1) exhibit no spectrally resolved vibronic structure, which is in contrast with the emission spectrum of anthracene. The spectra of $9 A-T_{2}$ and $9 A-T_{3}$ seem to contain a second spectral component producing a broad shoulder at the high-energy side of the spectrum above about $20000 \mathrm{~cm}^{-1}$. The maximum of the predominant emission band is located at about $17000 \mathrm{~cm}^{-1}$ for both compounds. All fluorescence spectra are independent of the excitation energy. Also fluorescence excitation spectra at room temperature were recorded. They are not plotted because they correspond to the absorption spectra and are independent of the detection energy.

Temperature dependent measurements between $T=300 \mathrm{~K}$ and $T=4.2 \mathrm{~K}$ were performed to get more information about the fluorescence behaviour of the substituted oligothiophenes. The spectra at $T=4.2 \mathrm{~K}$ in frozen $n$-hexane solution are distinctly different from those at room temperature. Obviously a clear chain length dependence exists (fig. 2); the emission bands monotonically shift to lower energies for increasing number of thiophene units. For $9 A-T_{2}$ and $9 \mathrm{~A}-\mathrm{T}_{3}$ the observed fluorescence is situated in the same spectral region as the broad shoulder on the high-energy side of the respective room temperature spectrum in fig. 1.

In addition, fig. 2 shows the fluorescence excitation spectra at $T=4.2 \mathrm{~K}$. The detection energy corresponds to the maximum position of the respective emission spectra. Detection at different energies results in the same fluorescence excitation spectra. The spectral composition is the same as in the absorption spectra at $T=295 \mathrm{~K}$ (see fig. 1), whereas the relative intensities of the bands do not reflect the true ones but are modified due to wavelength dependent diffraction of the excitation light in the frozen solution. The vibronic substructure is more pronounced for the absorption at low temperature. For $9 \mathrm{~A}-\mathrm{T}_{1}$ the substructure within the low energy band is due to the vibronic progression of the anthracene $S_{0}-S_{1}$ transition, whereas for $9 A-T_{4}$ the vibronic structure is indicative for the oligothiophene $\mathrm{T}_{4}$ absorption.

The fluorescence spectra of $9 \mathrm{~A}-\mathrm{T}_{2}$ and $9 \mathrm{~A}-\mathrm{T}_{3}$ at intermediate temperatures are plotted in fig. 3 . Clearly

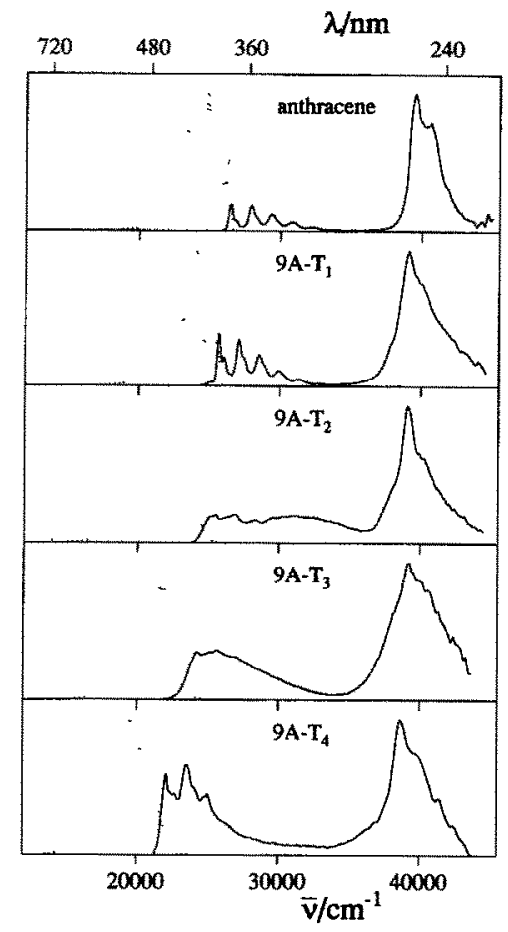

Fig. 2. Spectra of $9 \mathrm{~A}-T_{n}$ in $n$-hexane at $T=4.2 \mathrm{~K}$ : (-) relative absorption, detected via fluorescence excitation $\left(\lambda_{\text {det }}=\right.$ maximum of emission spectra $),(\cdots)$ fluorescence emission, $\lambda_{\mathrm{ex}}=257 \mathrm{~nm}$.

two spectral contributions to the fluorescence in the following denoted as "blue" and "red" components can be distinguished, whose relative intensities change monotonically as a function of temperature. In frozen $n$-hexane solution between $T=4.2$ and $T \approx 120 \mathrm{~K}$ for both compounds only the blue emission component is observed. Between $T \approx 120$ and $T \approx 130 \mathrm{~K}$ additionally the red component grows and finally dominates the spectrum for $T \geqslant 150 \mathrm{~K}$. The interchange takes place in the temperature region below the melting point of the solvent $n$-hexane $(T=178 \mathrm{~K})$. The red emission of the compounds $9 \mathrm{~A}-\mathrm{T}_{2}$ and $9 \mathrm{~A}-\mathrm{T}_{3}$ has the same maximum energy at about $17000 \mathrm{~cm}^{-1}$ whereas the blue emission is centred at $22700 \mathrm{~cm}^{-1}$ for $9 \mathrm{~A}-\mathrm{T}_{2}$ and at $21300 \mathrm{~cm}^{-1}$ for $9 \mathrm{~A}-\mathrm{T}_{3}$ (see fig. 4). From the temperature dependent spectral evolution the assumption given above is supported that also the fluorescence in liquid solution at room temperature is composed of two components.

Furthermore the properties of the two fluorescence 


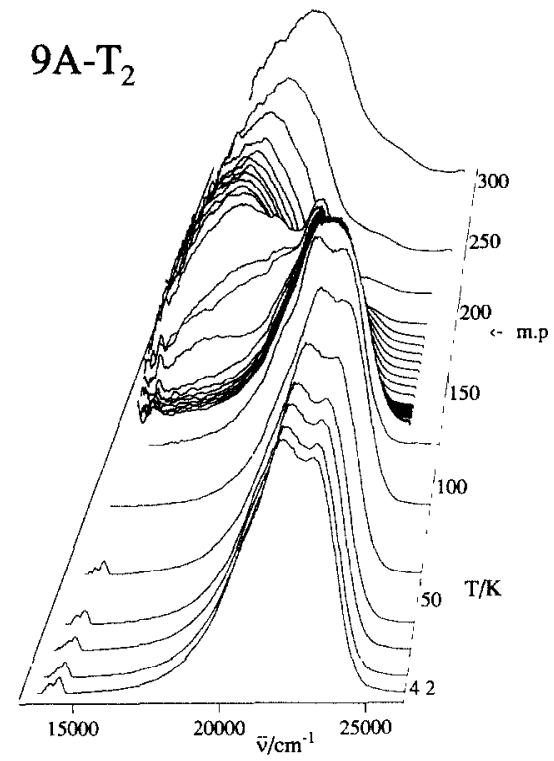

(a)

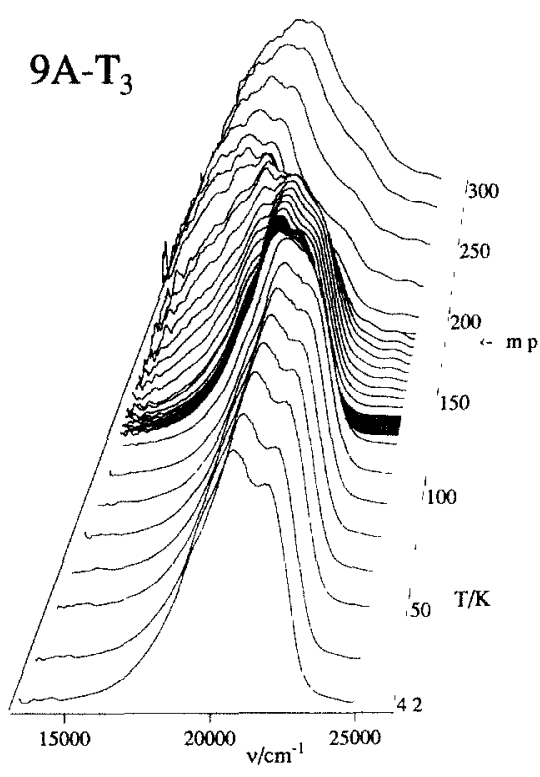

(b)

Fig. 3. Temperature dependence of the fluorescence spectra of (a) $9 A-T_{2}$ and (b) $9 A-T_{3}$ normalized to the same maximum intensity (m.p. = melting point of the solvent $n$-hexane, $T=178 \mathrm{~K}$ ).

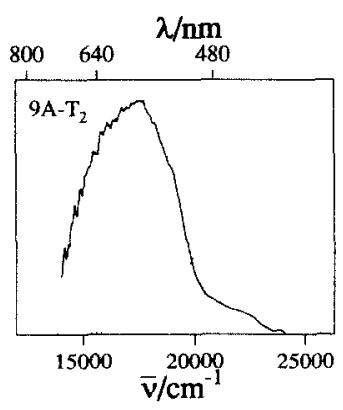

(a)

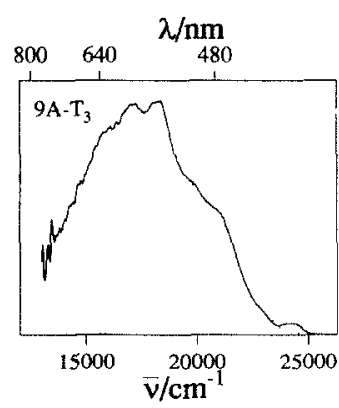

(b)
Fig. 4. Comparison of the fluorescence spectra of (a) $9 \mathrm{~A}-\mathrm{T}_{2}$ and (b) $9 \mathrm{~A}_{-} \mathrm{T}_{3}$ at (-) $T=180 \mathrm{~K}$ and $(\cdots) T=4.2 \mathrm{~K}$, normalized to the same maximum intensity ( $R=$ Raman line).

components of $9 \mathrm{~A}-\mathrm{T}_{2}$ and $9 \mathrm{~A}-\mathrm{T}_{3}$ were distinguished by time resolved measurements. As an example time resolved spectra and transients observed for $9 \mathrm{~A}-\mathrm{T}_{2}$ at $T=180 \mathrm{~K}$ are shown in fig. 5 . The quasi-cw spectrum in fig. 5 a detected in the time interval $[0 . .9000 \mathrm{ps}]$ closely resembles the emission spectrum given in fig. $4 a$, whereas in fig. $5 \mathrm{~b}$ the blue and red fluorescence components can be distinguished after ps laser pulse excitation upon detection in the largely differing time intervals of $[0 \ldots 25 \mathrm{ps}]$ and [ $500 \ldots 9000 \mathrm{ps}]$, dotted and solid curves in fig. $5 \mathrm{~b}$, respectively. The transient of the blue emission component reveals an immediate rise and a fast decay time of $27 \pm 5$ ps (fig. $5 \mathrm{c}$, right). The transient of the red emission instead shows a much slower decay time of $950 \pm 50 \mathrm{ps}$ and a measurable rise time of about $30 \pm 6$ ps (fig. $5 \mathrm{c}$, left). For the compound $9 \mathrm{~A}-\mathrm{T}_{3}$ an equivalent temporal and spectral behaviour is found. At $T=180 \mathrm{~K}$ for the blue emission component a decay time of $40 \pm 10$ ps is measured, for the red component a rise time of about $50 \pm 10 \mathrm{ps}$ and a decay time of $670 \pm 30$ ps.

For $9 \mathrm{~A}-\mathrm{T}_{1}$ the fluorescence spectra also revealed two emission components which however could only be differentiated by time resolved spectroscopy. $9 \mathrm{~A}-$ $\mathrm{T}_{4}$ shows only one emission component, which corresponds to the blue one, observed for the other anthryl-oligothiophenes. The decay times for all compounds $9 \mathrm{~A}-\mathrm{T}_{n}(n=1-4)$ are listed in table 1 together with those of the reference molecules $\mathrm{T}_{n}(n=2-4)$ and anthracene.

To compare the blue and red fluorescence components of $9 A-T_{2}$ and $9 A-T_{3}$ quantitatively the relative 


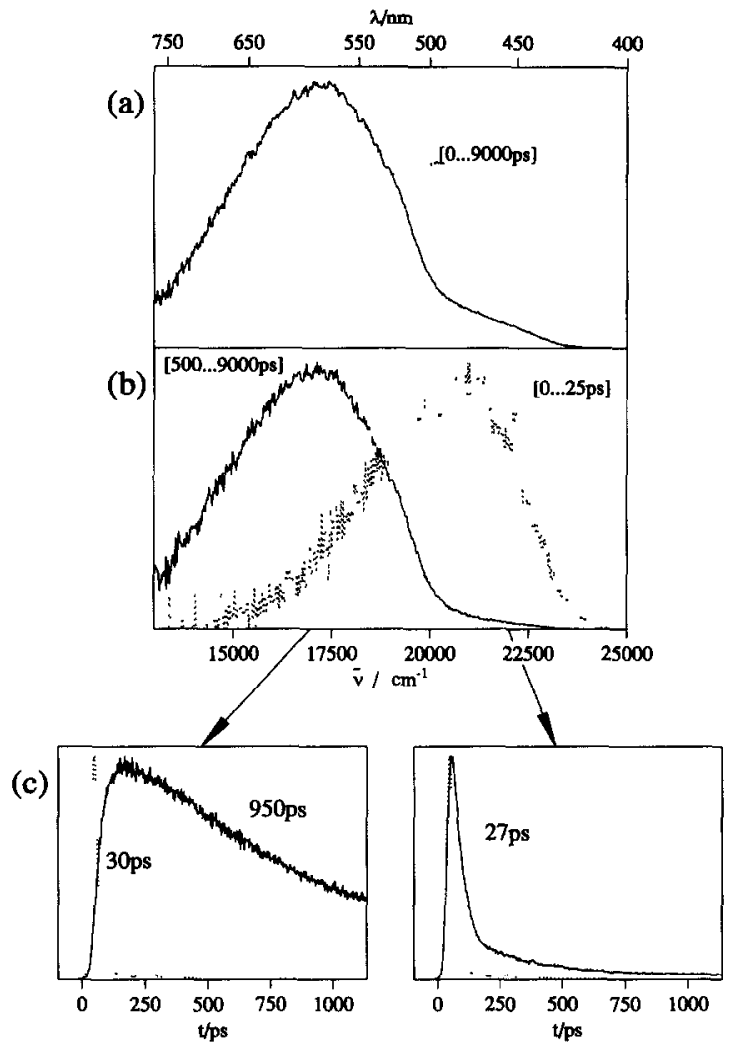

Fig. 5. Time resolved fluorescence measurements of $9 A-T_{2}$ in $n$ hexane at $T=180 \mathrm{~K}$. (a) $\mathrm{cw}$ reference spectrum. (b) Time resolved emission spectra detected in two different time intervals after picosecond pulse excitation: $(\cdots)$ between 0 and 25 ps; ( $\longrightarrow$ ) between 500 and 9000 ps. (c) Fluorescence transients detected in the red and blue emission bands, at 17000 and at $22000 \mathrm{~cm}^{-1}$, respectively, $(\cdots)$ pulse response of the apparatus.

fluorescence quantum yields were extracted from the time resolved emission spectra. The time resolved investigation reveals a similar fluorescence behaviour for $9 \mathrm{~A}-\mathrm{T}_{1}$, but here the extraction of the quantum yields was not possible due to the spectral overlap of the two fluorescence components, which both carry low fluorescence quantum yields. The quantum yields of the blue and red fluorescence components $\Phi_{b}$ and $\Phi_{\mathrm{r}}$ of $9 \mathrm{~A}-\mathrm{T}_{2}$ and $9 \mathrm{~A}-\mathrm{T}_{3}$ are plotted as a function of temperature in fig. 6 together with the overall values $\Phi_{\mathrm{f}}$. For both compounds $\Phi_{\mathrm{f}}$ is decreasing with increasing temperatures, from $68 \%$ at $T=4.2 \mathrm{~K}$ to $10 \%$ at $T=295 \mathrm{~K}\left(9 \mathrm{~A}-\mathrm{T}_{2}\right)$ and from $40 \%$ at $T=4.2 \mathrm{~K}$ to $5 \%$ at $T=295 \mathrm{~K}\left(9 \mathrm{~A}-\mathrm{T}_{3}\right)$.

At intermediate temperatures close to the melting
Table 1

Measured decay times $\tau$ and fluorescence quantum yields $\phi$ for 9A-T $T_{n}(n=1-4)$ investigated at $T=295 \mathrm{~K}$ and $T=180 \mathrm{~K}$, and for reference compounds oligothiophenes and anthracene. The fluorescence of $\mathrm{T}_{2}$ was fitted biexponentially. For 9A-T $(n=1-$ 3 ) the decay times and the individual fluorescence quantum yields of blue and red components are given (all values are accurate within 10\%)

\begin{tabular}{lcccc}
\hline & $\begin{array}{l}\tau^{295} \\
(p s)\end{array}$ & $\begin{array}{l}\tau^{180} \\
(p s)\end{array}$ & $\begin{array}{l}\phi^{295} \\
(\%)\end{array}$ & $\begin{array}{c}\phi^{180} \\
(\%)\end{array}$ \\
\hline $\mathrm{T}_{2}$ & $40 / 100$ & $75 / 170$ & 1.0 & 1.9 \\
$\mathrm{~T}_{3}$ & 150 & 270 & 5.6 & 9.8 \\
$\mathrm{~T}_{4}$ & 500 & 610 & 16.1 & 19.4 \\
anthracene & 5400 & 7900 & 30 & 55 \\
9A-T & $<10$ & 50 & - & - \\
& 290 & 290 & 2.1 & 2.8 \\
9A-T & $<10$ & 27 & 0.6 & 1.2 \\
& 900 & 950 & 9.4 & 12.8 \\
9A-T & 10 & 40 & 0.3 & 1.2 \\
& 650 & 670 & 5.2 & 8.8 \\
9A-T & 180 & 200 & 4.1 & 8.1 \\
\hline
\end{tabular}

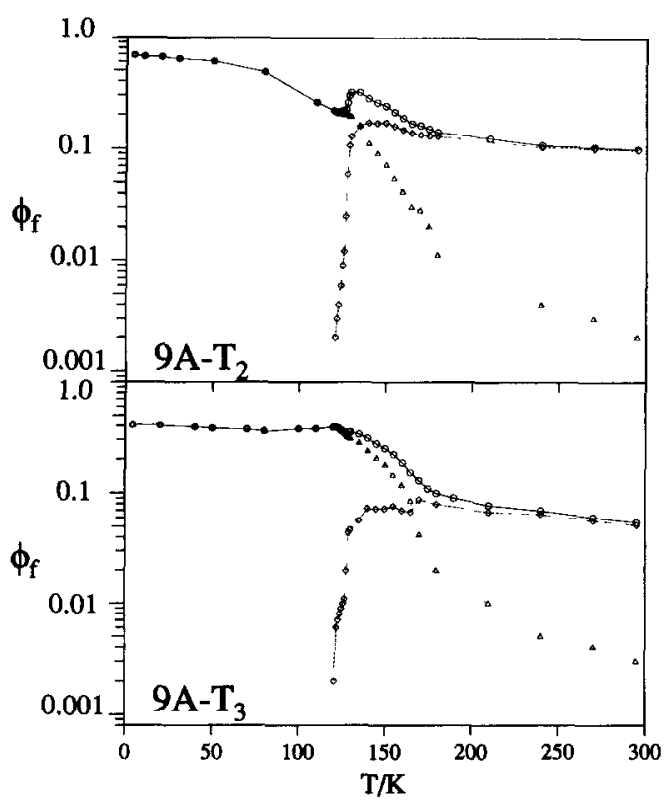

Fig. 6. Fluorescence quantum yields of $9 A-T_{2}$ and $9 A-T_{3}$ as a function of temperature: overall (circles), blue (triangles) and red (rhomboids) emission, respectively.

point of the solvent a stepwise decrease of $\Phi_{\mathrm{r}}$ is observed. A more pronounced decrease of $\Phi_{\mathrm{b}}$ and a corresponding increase of $\Phi_{\mathrm{r}}$ occurs for both compounds $9 \mathrm{~A}-\mathrm{T}_{2}$ and $9 \mathrm{~A}-\mathrm{T}_{3}$. 


\section{Discussion}

\subsection{Composition of the $9 A-T_{n}$ absorption spectra}

In good approximation the absorption spectra of the compounds $9 \mathrm{~A}-\mathrm{T}_{n}(n=1-4)$ can be regarded as a superposition of the absorption of the molecules anthracene and oligothiophene. There are no additional bands due to mixed electronic states as seen in 9 -anthryl-polyenes $[6,15]$. With respect to the $a b$ sorption spectra of anthracene and oligothiophene the observed bands are red-shifted and broadened. This is an indication for the coupling between the two molecular subunits.

For $9 \mathrm{~A}-\mathrm{T}_{1}$ and $9 \mathrm{~A}-\mathrm{T}_{2}$ the lowest energy absorption band can be clearly assigned to the anthracene $S_{0}-S_{1}$ transition. For $9 \mathrm{~A}-\mathrm{T}_{3}$ the $\mathrm{S}_{0}-S_{1}$ absorption bands of the subunits anthracene and terthiophene are overlapping in the same spectral region. For $9 \mathrm{~A}-\mathrm{T}_{4}$ the lowest energy absorption band is due to the thiophene moiety $\mathrm{T}_{4}$. As will be shown in the following this interchange in character (from anthracene- to oligothiophene-type) has important consequences for the fluorescence properties of the 9-anthryloligothiophenes.

\subsection{Chain length dependence and fluorescence energies at $T=4.2 \mathrm{~K}$}

At $T=4.2 \mathrm{~K}$ there is a systematic fluorescence redshift with increasing oligothiophene chain length. Because the fluorescence sets in at energies corresponding to the red edge of the respective lowest absorption band, which changes its character from anthraceneto oligothiophene-type upon increase of the oligothiophene chain length between $9 \mathrm{~A}-\mathrm{T}_{1}$ and $9 \mathrm{~A}-\mathrm{T}_{4}$, also the fluorescence character is expected to change. In order to test this idea a spectral analysis of the measured fluorescences (fig. 2) has been performed. In fig. 7 the energetic position of the 0.0 emission bands of $9 A-\mathrm{T}_{n}(n=1-4)$ are plotted against the inverse number of double bonds $(1 / k ; k=2 n)$ and compared with those of unsubstituted oligothiophenes $\mathrm{T}_{n}(n=2-5)$. For $\mathrm{T}_{n}$ and several other unsubstituted chain molecules (e.g. polyenes [16,17], cyanines [18] and rylenes [19]) such a plot reveals a linear relationship, which is described by the extended FEMO model [17]. The measured $n$-depen-

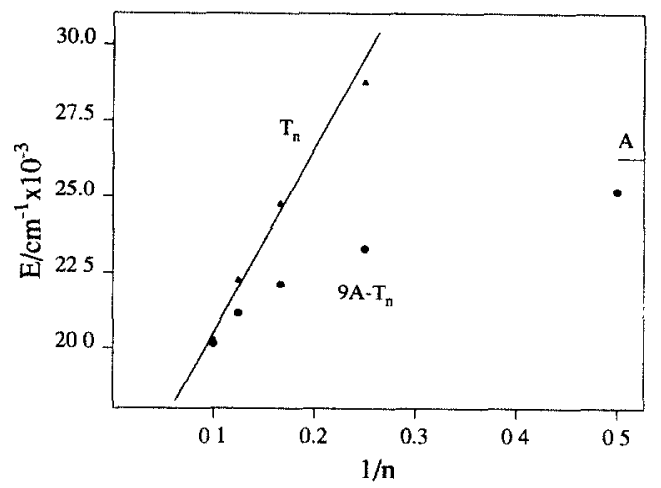

Fig. 7. Energetic position (0.0) of the emission bands of $9 A-T_{n}$ (C) and unsubstituted oligothıphenes $T_{n}(\Delta)$ as function of the inverse number of double bonds $1 / k=1 / 2 n$; a reference value for anthracene is indicated (A).

dence for $9 \mathrm{~A}-\mathrm{T}_{n}$ clearly deviates from this common behaviour. In fig. 7 , however, it is obvious that the $9 \mathrm{~A}-\mathrm{T}_{n}$ curve on the one hand approaches asymptotically that of $\mathrm{T}_{n}$ whereas on the other hand the measured energetic position of $9 A-T_{1}$ is close to that of unsubstituted anthracene (also given in fig. 7 ). This confirms that also the fluorescence emission at $T=4.2$ $\mathrm{K}$ in the compounds $9 \mathrm{~A}-\mathrm{T}_{n}$ changes its character from anthracene-type to oligothiophene-type upon varying $n$ between $n=1$ and $n=4$.

\subsection{Dual fluorescence of $9 A-T_{n}(n=1-3)$ at higher temperatures}

Dual fluorescence [20] is reported for donor/acceptor-substituted aryls and biaryls, e.g. dimethylaminobenzonitrile $[21,22]$ or bianthryl $[3,4]$ in polar solvents and has been explained by a photoinduced creation of a charge separated excited state. The charge separation in the excited state is related to a discrete twisted molecular conformation (TICT, twisted intramolecular charge transfer $[23,24])$, which is stabilized by the polar solvent molecules. In nonpolar solvents typical TICT molecules show merely one fluorescence from the locally excited state [25]. Only for a few molecules with strong donor groups (dimethylamino, pyrrolidino, etc.) also in nonpolar solvents an additional emission assigned to a TICT state was found [24].

In the molecules $9 \mathrm{~A}-\mathrm{T}_{n}$, however, strong donor 
groups are not present and the dual fluorescence occurs in the nonpolar solvent $n$-hexane. Quantitative energetic considerations for the possible formation of an excited state with complete charge transfer (TICT) taking into account Coulomb and solvent interaction [25] in different solvents have been made using the redox potentials of both chromophores anthracene [26] and oligothiophene [27]. The result is that such CT formation would be clearly endothermic. Apparently a TICT state is not to be expected for the studied systems $9 \mathrm{~A}-\mathrm{T}_{n}(n=1-3)$ in the nonpolar solvent $n$-hexane. (These considerations do not exclude partial CT character of the excited state in polar environment.)

From the experimental results it is obvious that the viscosity of the solvent is a crucial parameter for the appearance and the dynamics of the dual fluorescence of the compounds $9 A-T_{2}$ and $9 A-T_{3}$. The red component can be frozen in at low temperatures. Therefore we suggest that the essential parameter in our molecular systems, similarly as stated for other biaryl systems e.g. [ 1,2$]$, is the intramolecular torsional angle for motion about the chemical bond between the anthryl and the oligothiophene moieties. This means we introduce a one-dimensional effective potential in the torsional angle $\vartheta$ (fig. 8). Two competing forces determine the equilibrium angular position: the minimization of the $\pi$-system's delocalization energy tends to planarize the supermolecule

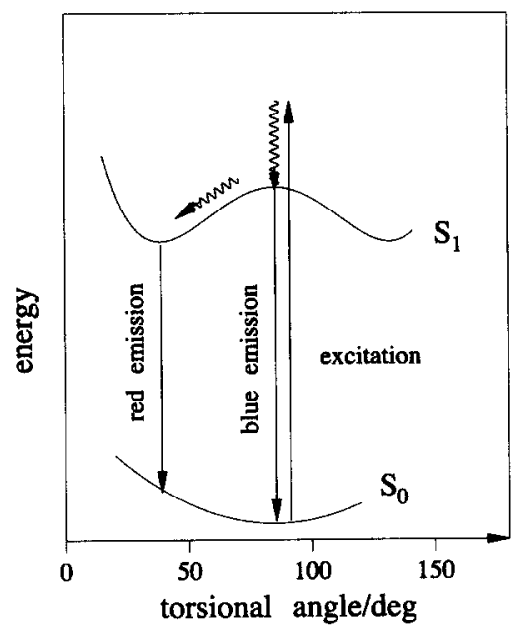

Fig. 8. Torsional potential of $9 \mathrm{~A}-\mathrm{T}_{n}$ and optical transitions, schematically. (towards $\vartheta=0^{\circ}$ ) whereas the sterical hindrance of the ortho-hydrogen atoms favours a perpendicular conformation $\left(\vartheta=90^{\circ}\right)$. The considerations of the frontier orbitals of the two chromophores anthracene and oligothiophene show a higher degree of $\pi$-resonance between the two subunits in the excited state. This enlarged $\pi-\pi$-interaction planarizes the supermolecule in the excited state with respect to the ground state conformation.

For the ground state X-ray diffraction measurements of crystalline $9 A-T_{1}$ reveal a large dihedral angle of $\vartheta=72^{\circ}$ between anthracene and thiophene [12]. From MNDO/AM1 calculations for isolated molecules $9 \mathrm{~A}-\mathrm{T}_{1}$ and $9 \mathrm{~A}-\mathrm{T}_{2}$ in the gas phase a ground state torsional potential with a - rather flat - minimum at about $\vartheta=90^{\circ}$ is obtained [28], which is in agreement with the small degree of coupling between the two chromophores. In liquid nonpolar solution the molecular conformation should be very similar. As a hint for a reduced torsional angle in the excited state preliminary AM1 calculations yield an average torsional angle of about $\vartheta=62^{\circ}$ for $9 \mathrm{~A}-\mathrm{T}_{1}$ and about $\vartheta=54^{\circ}$ for $9 \mathrm{~A}-\mathrm{T}_{2}$.

As indicated in fig. 8 we expect competing relaxation pathways in the optically excited state: first, direct relaxation to the ground state while maintaining the initial molecular conformation, second, fast relaxation along $\vartheta$ within the excited state towards the effective minimum angle and subsequent relaxation from that conformation. According to this model the blue and red emissions are assigned to different conformations of the molecule, which are coupled dynamically. The competing relaxation pathways depend on the solvent's viscosity and consequently its temperature.

The temperature dependent measurements show that the blue fluorescence quantum yields of $9 \mathrm{~A}-\mathrm{T}_{2}$ and $9 \mathrm{~A}-\mathrm{T}_{3}$ are quite high in the temperature range below $T \approx 120 \mathrm{~K}$, when the intramolecular torsion between anthracene and oligothiophene is frozen in. For $T>120 \mathrm{~K}$ the red emission component appears, since the torsion between the two chromophores is made possible, but the overall fluorescence quantum yield decreases due to additional nonradiative relaxation processes. 


\section{Conclusion}

In this paper, absorption and fluorescence spectra of anthryl-oligothiophenes $9 \mathrm{~A}-\mathrm{T}_{n}$ have been characterized as a function of chain length $(n=1-4)$ and temperature. The emission spectra reveal a dual fluorescence for $9 \mathrm{~A}-\mathrm{T}_{1}, 9 \mathrm{~A}-\mathrm{T}_{2}$ and $9 \mathrm{~A}-\mathrm{T}_{3}$ at $T \geqslant 120 \mathrm{~K}$ and a dynamical coupling of the two fluorescence components in the nonpolar $n$-hexane solution. This behaviour is explained by an intramolecular torsional motion between the two molecular subunits anthracene and oligothiophene. The fact that the dual fluorescence for $9 A-T_{1}$ is very weak and does not exist for $9 \mathrm{~A}-\mathrm{T}_{4}$ can be described by the change of the predominant character of the lowest excited singlet state from anthracene- to oligothiophene-type. The present results are important for the understanding of the intramolecular energy transfer currently studied in 9-anthryl-oligothiophenes additionally substituted by an acceptor molecule.

\section{Acknowledgement}

We gratefully acknowledge financial support from the Deutsche Forschungsgemeinschaft (SFB 329).

\section{References}

[1] D.W. Werst, A.M. Brearley, W.R. Gentry and P.F. Barbara, J. Am. Chem. Soc. 109 (1987) 32.

[2] R. Wortmann, S. Lebus, K. Elich, S. Assar, N. Detzer and W. Liptay, Chem. Phys. Letters 198 (1992) 220.

[3] F. Schneider and E. Lippert, Ber. Bunsenges. Physik. Chem. 72 (1968) 1155.

[4] W. Rettig and M. Zander, Ber. Bunsenges. Physik. Chem. 87 (1983) 1143.

[5] T.J. Kang, W. Jarzeba, P.F. Barbara and T. Fonseca, Chem. Phys. 149 (1990) 81 .
[6] N. Holl, P. Emele, H. Port, H.C. Wolf, H. Strobel, T. Kesmarszky and F. Effenberger, Chem. Phys. Letters 205 (1993) 25.

[7] F. Effenberger, H. Schlosser, P. Băuerle, St. Maier, H. Port and H.C. Wolf, Angew. Chem. 100 (1989) 274.

[8] G. Blessing, N. Holl, H. Port, H.C. Wolf, F. Effenberger, Th. Kesmarszky and H. Schlosser, Mol. Cryst. Liquid Cryst. $183(1990) 21$.

[9] N. Holl, Dissertation, Universität Stuttgart (1993).

[10] P. Garcia, J.M. Pernaut, P. Hapiot, V. Wintgens, P. Valat, F. Garnier and D. Delabouglise, J. Phys. Chem. 97 (1993) 513.

[11] P. Bäuerle, F. Würthner, G. Götz and F. Effenberger, Synthesis, in press.

[12] F. Würthner, Dissertation, Universität Stuttgart (1993).

[13] M. Wiechmann, H. Port, W. Frey, F. Lärmer and T. Elsässer, J. Phys. Chem. 95 (1991) 1918.

[14] S. Ates and A. Yildiz, J. Chem. Soc. Faraday Trans. I 79 (1983) 2853.

[15] P. Gribi, G. Isenmann, E. Sigmund, G. Quapil. N. Holl and H. Port, J. Chem. Phys. 98 (1993) 7696.

[16] B.S. Hudson, B.E. Kohler and K. Schulten, in: Excited states, Vol. 6, ed. E.C. Lim (Academic Press, New York, 1982).

[17] J.N. Murrell, The theory of electronic spectra of organic molecules (Methuen, London, 1963).

[18]H.D. Försterling and H. Kuhn, Moleküle und Molekülanhäufungen (Springer, Berlin, 1983).

[19] S. Raisch, Diplomarbeit, Universität Stuttgart (1992).

[20] J.B. Birks, Photophysics of aromatic molecules (WilcyInterscience, New York, 1970).

[21 ] E. Lippert, W. Lüder and H. Boos, in: Advances in molecular spectroscopy, ed. A. Mangini (Pergamon Press, Oxford, $1962)$ p. 443.

[22] O. Kajimoto, H. Yokoyama, Y. Ooshima and Y. Endo, Chem. Phys. Letters 179 (1991) 455.

[23] Z.R. Grabowski and J. Dobkowski, Pure Appl. Chem. 55 (1983) 245.

[24] W. Rettig, Angew. Chem. 98 (1986) 969.

[25] M. Zander and W. Rettig, Chem. Phys. Letters 110 (1984) 602.

[26] M. Julliard and M. Chanon, Chem. Rev. 83 (1983) 425; E.S. Pysh and N.C. Yang, J. Am. Chem. Soc. 85 (1963) 2125 .

[27] A.F. Diaz, J. Crowley, J. Bargon, G.P. Gardini and J.B. Torrance, J. Electroanal. Chem. 121 (1981) 355; D. Jones, M. Guera, L. Favaretto, A. Modelli, M. Fabrizio and G. Distefano, J. Phys. Chem. 94 (1990) 5761.

[28] K. Elsässer, Diplomarbeit, Universität Stuttgart (1992). 